\title{
Clinical utility gene card for: Alport syndrome
}

\author{
Jens Michael Hertz ${ }^{\star,}$, Mads Thomassen ${ }^{1}$, Helen Storey ${ }^{2}$ and Frances Flinter ${ }^{3}$ \\ European Journal of Human Genetics (2012) 20, doi:10.1038/ejhg.2011.237; published online 14 December 2011
}

\section{DISEASE CHARACTERISTICS}

1.1 Name of the disease (synonyms)

Alport syndrome.

\subsection{OMIM\# of the disease}

301050 (X-linked form), 203780 (autosomal recessive form), and 104200 (autosomal dominant form).

1.3 Name of the analysed genes or DNA/chromosome segments COL4A5, COL4A4, and COL4A3.

\subsection{OMIM\# of the gene(s)}

303630 (COL4A5), 120070 (COL4A3), 120131 (COL4A4).

\subsection{Mutational spectrum}

COL4A5: missense mutations ( $\sim 41 \%$ of the mutations; $\sim 85 \%$ of which are glycine substitutions in the conserved Gly-Xaa-Yaa repeat sequence in the collagenous domain of the alpha5(IV)-chain); splice site mutations $(\sim 16 \%)$; frame shifts (small deletions, small insertions/ duplications) ( $\sim 22 \%)$; nonsense mutations ( $\sim 6 \%)$; large deletions, insertions/duplications, and other larger structural rearrangements (inversions and translocations) $(\sim 15 \%)$. More than 685 mutations are known.

COL4A4: missense mutations ( $\sim 50 \%$; $\sim 75 \%$ of which are glycine substitutions in the conserved Gly-Xaa-Yaa repeat sequence in the collagenous domain of the alpha4(IV)-chain); frame shifts (small deletions, small insertions/duplications) $(\sim 27 \%)$; splice site mutations ( $\sim 13 \%)$; nonsense mutations $(\sim 9 \%)$, and larger deletions $(\sim 1 \%)$. More than 56 mutations are known.

COL4A3: missense mutations ( $\sim 45 \%$ of the mutations; $\sim 85 \%$ of which are glycine substitutions in the conserved Gly-Xaa-Yaa repeat sequence in the collagenous domain of the alpha3(IV)chain); frame shifts (small deletions, small insertions/duplications) ( $\sim 21 \%)$; nonsense mutations $(\sim 15 \%)$; splice site mutations $(\sim 15 \%)$, and larger deletions $(\sim 4 \%)$. More than 73 mutations are known.

See:

HGMD (http://www.hgmd.org).

Alport LOVD databases (COL4A5, COL4A3, COL4A4)

(https://grenada.lumc.nl/LOVD2/COL4A/home.php?action=switch_db). ARUP Alport (COL4A5) database (www.arup.utah.edu/database/ ALPORT/ALPORT_welcome.php).

\subsection{Analytical methods}

Direct sequencing of genomic exonic DNA, including flanking intronic sequences. Reverse transcriptase PCR analysis of mRNA extracted from cultured skin fibroblasts or hair roots for deep intronic mutations causing RNA splicing aberrations (X-linked form). Multiplex ligation-dependent probe amplification (MLPA) for detection of submicroscopic deletions and duplications. Conventional cytogenetic analysis for detection of structural chromosome rearrangements. Next-generation sequencing is a promising new technique for a genetic heterogenous disorder like Alport syndrome.

\subsection{Analytical validation}

Confirmation in an independent biological sample from the index case or an affected relative. Single exon deletions and duplications should be confirmed with another technique.

\subsection{Estimated frequency of the disease}

(incidence at birth ('birth prevalence') or population prevalence) The most widely used estimate of the prevalence of Alport syndrome is 1:5000, based on the finding of about 300 cases in Utah and southern Idaho in a population of 1500000 people. ${ }^{1}$ The incidence of Alport syndrome was found to be 1:53000 in Finland, ${ }^{2}$ and 1:17 000 in southern Sweden. ${ }^{3}$ The inheritance is $\mathrm{X}$-linked in $\sim 85 \%$ of the families, autosomal recessive in $\sim 15 \%$, and autosomal dominant in very few families.

1.9 If applicable, prevalence in the ethnic group of the investigated person

Not applicable.

\subsection{Diagnostic setting}

\begin{tabular}{lcc}
\hline & Yes & No \\
\hline A. (Differential) diagnostics & $\bigotimes$ & $\square$ \\
B. Predictive testing & $\bigotimes$ & $\square$ \\
C. Risk assessment in relatives & $\bigotimes$ & $\square$ \\
D. Prenatal & $\bigotimes$ & $\square$
\end{tabular}

\section{Comment:}

Mutation analysis is mainly used for confirmation of a clinical diagnosis and in relation to genetic counselling. Prenatal diagnosis and pre-implantation genetic diagnosis are available for families with a known mutation. In families with the X-linked form of

\footnotetext{
${ }^{1}$ Department of Clinical Genetics, Odense University Hospital, Sdr. Boulevard 29, DK-5000 Odense C, Denmark; ${ }^{2}$ DNA Laboratory, GSTS Pathology, Guy's Hospital, London SE1 9RT, UK; ${ }^{3}$ Department of Clinical Genetics, Guy's Hospital, London SE1 9RT, UK

${ }^{*}$ Correspondence: Professor JM Hertz, Department of Clinical Genetics, Odense University Hospital, Sdr. Boulevard 29, DK-5000 Odense C, Denmark. Tel: +45 6541 3191 ; Fax: +45 6541 4875; E-mail: Jens.Michael.Hertz@ouh.regionsyddanmark.dk
} 
the disease, prenatal diagnosis is performed only after sex determination, when the foetus is male.

\section{TEST CHARACTERISTICS}

\begin{tabular}{|c|c|c|c|c|}
\hline & \multicolumn{2}{|c|}{ Genotype or disease } & \multirow{2}{*}{$\begin{array}{l}\text { A: true positives } \\
\text { B: false positives }\end{array}$} & \multirow{2}{*}{$\begin{array}{l}\text { C: false negative } \\
\text { D: true negative }\end{array}$} \\
\hline & Present & Absent & & \\
\hline \multicolumn{5}{|l|}{ Test } \\
\hline \multirow[t]{2}{*}{ Positive } & A & B & Sensitivity: & $A /(A+C)$ \\
\hline & & & Specificity: & $D /(D+B)$ \\
\hline \multirow[t]{2}{*}{ Negative } & C & $\mathrm{D}$ & Positive predictive value: & $A /(A+B)$ \\
\hline & & & Negative predictive value: & $\mathrm{D} /(\mathrm{C}+\mathrm{D})$ \\
\hline
\end{tabular}

\subsection{Analytical sensitivity}

(proportion of positive tests if the genotype is present)

COL4A5: sequencing of genomic DNA and MLPA: $>95 \%$.

COL4A3 and COL4A4: sequencing of genomic DNA and MLPA: probably $>95 \%$.

Sequence variants of unknown significance may affect the sensitivity.

\subsection{Analytical specificity}

(proportion of negative tests if the genotype is not present)

COL4A5: Sequencing of genomic DNA and MLPA: $>95 \%$.

COL4A3 and COL4A4: Sequencing of genomic DNA and MLPA: $>95 \%$.

\subsection{Clinical sensitivity}

(proportion of positive tests if the disease is present)

The clinical sensitivity can be dependent on variable factors such as age or family history. In such cases, a general statement should be given even if quantification can only be made on a case-by-case basis.

$\mathrm{X}$-linked form: highly dependent on fulfillment of the clinical criteria for Alport syndrome ${ }^{4}$ and may be supported by immunohistochemical findings in a kidney and/or skin biopsy. Above $80 \%$ in families fulfilling three or more diagnostic criteria. Higher in families with obvious $\mathrm{X}$-linked inheritance, and higher in males than in females. ${ }^{5}$

Autosomal recessive form: highly dependent on fulfilment of the clinical criteria for Alport syndrome ${ }^{4}$ and may be supported by abnormal immunohistochemical findings in a kidney biopsy, and normal immunohistochemical findings in a skin biopsy. Above $80 \%$ in families fulfilling three or more diagnostic criteria. Higher in families with consanguinity, and in patients with a family history of thin basement membrane disease/benign familial hematuria.

\subsection{Clinical specificity}

\section{(proportion of negative tests if the disease is not present)}

The clinical specificity can be dependent on variable factors such as age or family history. In such cases, a general statement should be given even if quantification can only be made on a case-by-case basis.

X-linked form: almost $100 \%$ in males by the age of 20 years.

Autosomal recessive form: approaches $100 \%$ in males and females by the age of 20 years.

\subsection{Positive clinical predictive value}

(life-time risk to develop the disease if the test is positive)

$\mathrm{X}$-linked form: almost $100 \%$ with respect to end-stage renal disease (ESRD) in males, and $30-40 \%$ in females.
Autosomal recessive form: almost $100 \%$ for patients with homozygous or compound heterozygous COL4A3/COL4A4 mutations. Heterozygotes can have a completely normal phenotype, or a broad spectrum of symptoms ranging from thin basement membrane disease/benign familial hematuria (OMIM \#141200) to ESRD.

\subsection{Negative clinical predictive value}

(Probability not to develop the disease if the test is negative)

Assume an increased risk based on family history for a non-affected person. Allelic and locus heterogeneity may need to be considered.

Index case in that family had been tested:

X-linked form: almost $100 \%$.

Autosomal recessive form: almost $100 \%$ for patients with homozygous or compound heterozygous COL4A3/COL4A4 mutations. Heterozygotes can have a completely normal phenotype, or a broad spectrum of symptoms ranging from thin basement membrane disease/benign familial hematuria (OMIM \#141200) to ESRD.

Index case in that family had not been tested:

$\mathrm{X}$-linked form: unknown, but probably high.

Autosomal recessive form: unknown, but probably high.

\section{CLINICAL UTILITY}

3.1 (Differential) diagnosis: The tested person is clinically affected (To be answered if in 1.10 'A' was marked)

\subsubsection{Can a diagnosis be made other than through a genetic test?}

$\begin{array}{ll}\text { No } & \square \text { (continue with } \\ & 3.1 .4 \text { ) } \\ \text { Yes } & \square \\ & \text { Clinically } \\ \text { Imaging } \\ \text { Endoscopy } \\ \text { Biochemistry } \\ \text { Electrophysiology } \\ \text { Other (please } \\ \text { describe) }\end{array}$

$$
\begin{aligned}
& \text { Family history and a clinical examination including } \\
& \text { microscopic urine analysis, renal function study, }
\end{aligned}
$$
ophthalmoscopy, and audiometry.

Kidney biopsy: Electron microscopy for characteristic ultrastructural changes, and immunohistochemical staining for the alpha3-, alpha4-, and alpha5-chains of type IVcollagen. All three chains are absent in the glomerular basement membrane (GBM), Bowman's capsules and tubular basement membranes in 70-80\% of males with the X-linked form. In the autosomal forms of Alport syndrome all three chains are also absent from the GBM in most patients, but the alpha5-chain is present in Bowman's capsules and tubular basement membranes. Skin biopsy: Immunohistochemical staining for the alpha5(IV)-chain. Absent in the epidermal basement membrane in $70-80 \%$ of males with the X-linked form, and normal in patients with the autosomal forms. Recommended diagnostic procedure:

1. Family history and clinical examination.

2. Audiogram and eye examination by slit lamp ophthalmoscopy.

3. Skin biopsy for immunohistochemical staining for the alpha5(IV)-chain and RT-PCR analysis of mRNA from cultured fibroblasts, or, alternatively, direct sequencing of all 53 COL $4 A 5$ exons on genomic DNA.

4. MLPA analysis of genomic DNA for COL4A5 deletion/ duplication. ${ }^{6}$ 
(Continued)

5. Direct sequencing of $\mathrm{COL} 4 A 4$ and $\mathrm{COL} 4 A 3$ on genomic DNA in case of normal immunohistochemical staining for the alpha5(IV)-chain of the skin biopsy and no COL4A5 mutation or if the family history suggests autosomal inheritance (e.g. consanguineous parents). 6. Consider a kidney biopsy.

\subsubsection{Describe the burden of alternative diagnostic methods to the} patient.

Kidney biopsy confers a risk to the patient (bleeding, infection, and pain), and a skin biopsy will result in a tiny scar.

\subsubsection{How is the cost effectiveness of alternative diagnostic methods to be judged?}

Genetic testing is useful for confirming a clinical diagnosis, informing genetic counselling, and facilitating prenatal diagnosis and preimplantation genetic diagnosis. The three type-IV collagen genes, COL4A5, COL4A3, and COL4A4, are very large genes with 48-53 exons and a coding sequence of $>5000 \mathrm{bp}$. New technologies such as next generation sequencing may lower costs and turn-around time.

3.1.4 Will disease management be influenced by the result of a genetic test?

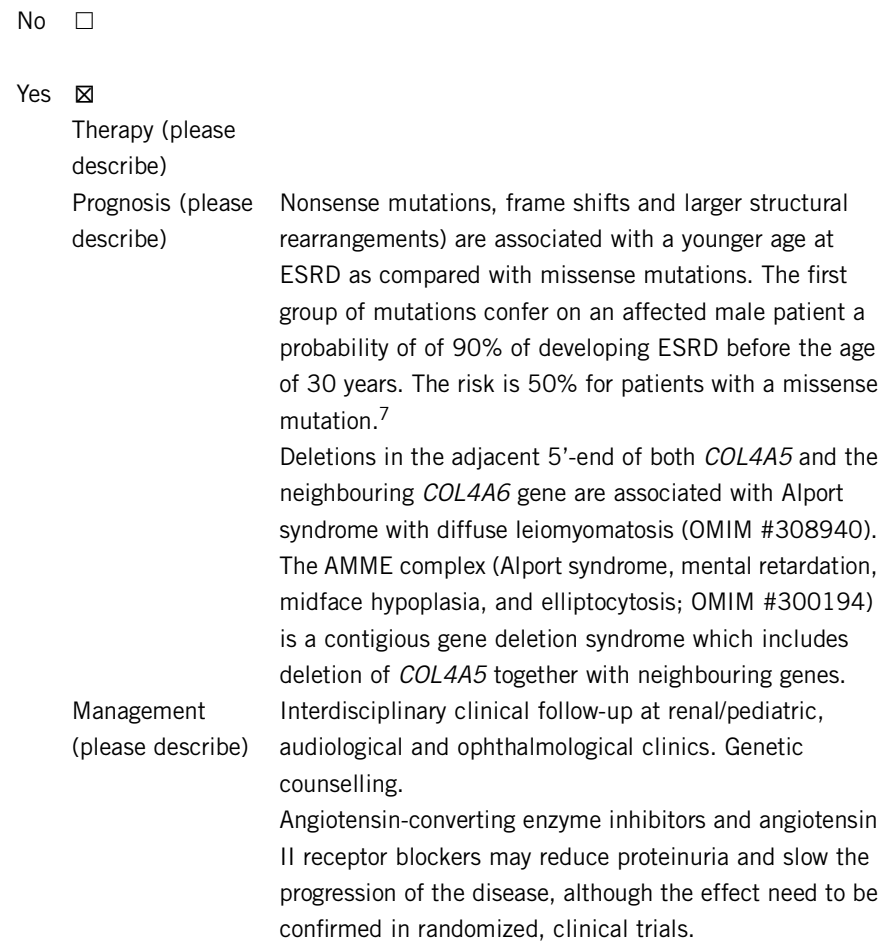

3.2 Predictive Setting: The tested person is clinically unaffected but carries an increased risk based on family history

(To be answered if in 1.10 ' $\mathrm{B}$ ' was marked)

\subsubsection{Will the result of a genetic test influence lifestyle and prevention?}

If the test result is positive (please describe):

If the test is positive, clinical follow-up and genetic counselling should be recommended. Careful management of blood pressure in a patient with a positive predictive test prolongs the functioning life of a patient's native kidneys and postpones the need for renal replacement therapy.

If the test result is negative (please describe):

Clinical follow-up is not relevant if the test is negative for a known familial mutation.

3.2.2 Which options in view of lifestyle and prevention does a person at-risk have if no genetic test has been done (please describe)? Avoiding potentially nephrotoxic drugs, and the same interdisciplinary surveillance programme comprising nephrological/pediatric, ophthalmological, and audiological examination as for those with a positive genetic test. The frequency depends on the clinical findings.

3.3 Genetic risk assessment in family members of a diseased person (To be answered if in 1.10 ' $\mathrm{C}$ ' was marked)

3.3.1 Does the result of a genetic test resolve the genetic situation in that family?

Yes. The risk assessment depends on the mode of inheritance; $\mathrm{X}$-linked, autosomal recessive or dominant.

3.3.2 Can a genetic test in the index patient save genetic or other tests in family members?

Yes. If a pathogenic mutation has been identified in the index patient, genetic counselling and testing for the familial mutation could be offered to relatives at risk. If a relative tests negative, then none of their offspring are at risk and they will not need to be tested.

\subsubsection{Does a positive genetic test result in the index patient enable a} predictive test in a family member?

Yes. Carrier testing and pre-symptomatic testing in relatives will be possible.

\subsection{Prenatal diagnosis}

(To be answered if in 1.10 'D' was marked)

3.4.1 Does a positive genetic test result in the index patient enable a prenatal diagnosis?

Yes, prenatal and pre-implantation diagnostics can be performed in the family, if requested.

\section{IF APPLICABLE, FURTHER CONSEQUENCES OF TESTING}

Please assume that the result of a genetic test has no immediate medical consequences. Is there any evidence that a genetic test is nevertheless useful for the patient or his/her relatives? (Please describe)

Genetic testing can be useful for the patient/family in relation to genetic counselling. Genetic testing of relatives can also be relevant in relation to selection of potential family donors for kidney transplantation.

\section{CONFLICT OF INTEREST}

The authors declare no conflict of interest.

\section{ACKNOWLEDGEMENTS}

This work was supported by EuroGentest2 (Unit 2: 'Genetic testing as part of health care'), a Coordination Action under FP7 (Grant Agreement Number 261469) and the European Society of Human Genetics.

1 Hasstedt SJ, Atkin CL: X-linked inheritance of Alport syndrome: family P revisited. Am J Hum Genet 1983; 35: 1241-1251.

2 Pajari $\mathrm{H}$, Kaariainen $\mathrm{H}$, Muhonen T, Koskimies O: Alport's syndrome in 78 patients: epidemiological and clinical study. Acta Paediatr 1996; 85: 1300-1306. 
3 Persson U, Hertz JM, Wieslander J, Segelmark M: Alport syndrome in southern Sweden. Clin Nephrol 2005; 64: 85-90.

4 Flinter FA, Cameron JS, Chantler C, Houston I, Bobrow M: Genetics of classic Alport's syndrome. Lancet 1988; 2: 1005-1007.

5 Hanson H, Storey H, Pagan J, Flinter F: The value of clinical criteria in identifying patients with X-linked Alport syndrome. Clin J Am Soc Nephrol. 2011; 6: 198-203.
6 Hertz JM, Juncker I, Marcussen N: MLPA and CDNA analysis improves COL4A4 mutation detection in X-linked Alport syndrome. Clin Genet 2008; 74: 522-530.

7 Jais JP, Knebelmann B, Giatras I et al: X-linked Alport syndrome: natural history in 195 families and genotype-phenotype correlations in males. J Am Soc Nephrol 2000; 11: 649-657. 